OPEN ACCESS

Edited by:

Zhi Sheng,

Virginia Tech, United States

Reviewed by:

Braden C. McFarland

University of Alabama at Birmingham,

United States

Victor C. Kok

Asia University, Taiwan

*Correspondence:

Eric Jakobsson

jake@illinois.edu

Specialty section:

This article was submitted to

Cancer Molecular Targets and

Therapeutics,

a section of the journal

Frontiers in Oncology

Received: 04 January 2019

Accepted: 01 April 2019

Published: 30 April 2019

Citation:

Ge W and Jakobsson E (2019)

Systems Biology Understanding of the

Effects of Lithium on Cancer.

Front. Oncol. 9:296.

doi: 10.3389/fonc.2019.00296

\section{Systems Biology Understanding of the Effects of Lithium on Cancer}

\author{
Weihao Ge ${ }^{1,2}$ and Eric Jakobsson ${ }^{1,2,3 *}$ \\ ${ }^{1}$ National Center for Supercomputing Applications, University of Illinois at Urbana-Champaign, Urbana, IL, United States, \\ ${ }^{2}$ Center for Biophysics and Computational Biology, University of Illinois at Urbana-Champaign, Urbana, IL, United States, \\ ${ }^{3}$ Department of Molecular and Integrative Physiology, University of Illinois at Urbana-Champaign, Urbana, IL, United States
}

Lithium has many widely varying biochemical and phenomenological effects, suggesting that a systems biology approach is required to understand its action. Multiple lines of evidence point to lithium as a significant factor in development of cancer, showing that understanding lithium action is of high importance. In this paper we undertake first steps toward a systems approach by analyzing mutual enrichment between the interactomes of lithium-sensitive enzymes and the pathways associated with cancer. This work integrates information from two important databases, STRING, and KEGG pathways. We find that for the majority of cancer pathways the mutual enrichment is statistically highly significant, reinforcing previous lines of evidence that lithium is an important influence on cancer.

Keywords: lithium, systems biology, biochemical pathways, biochemical networks, cancer, gsk3b, kinases, phosphotransferases

\section{INTRODUCTION}

\section{Clinical and Epidemiological Context for Lithium and Cancer}

By far the most common medical use of lithium is as a first line therapy for bipolar disorder, including associated depression as well as mania (1). A comprehensive review of the literature confirms that lithium is also effective against unipolar depression with unique anti-suicidal effectiveness, and may also be useful against cancer and neurodegenerative disease $(2,3)$.

One line of evidence for the possible use of lithium as an anticancer agent is epidemiological. A retrospective study showed that psychiatric patients undergoing lithium therapy for bipolar disorder had a much lower incidence of cancer than a matched group not receiving lithium therapy (4). More recent studies of similar design, one conducted nationwide across Sweden, and another across Taiwan, achieved the same result $(5,6)$. On the other hand another nationwide study, this time from Denmark, showed no correlation of lithium with colorectal adenocarcinoma (7). On closer look, the Denmark study does not contradict the Swedish study. The Swedish study also found that for the entire population lithium was not correlated with cancer incidence, but in addition found that bipolar individuals not treated with lithium had a higher incidence of cancer than the general population. Lithium-treated bipolar patients, on the other hand, had essentially the same cancer incidence as the general population.

One piece of experimental evidence for lithium's potential as a cancer therapeutic modality is that it was observed to inhibit prostate tumor growth (8), presumably through its ability to inhibit GSK3. A detailed study of molecular mechanisms by which lithium inhibition of GSK3beta inhibits proliferation of prostate tumor cells in culture was presented by Sun et al. (9). The work was subsequently extended to an animal model (10). A clinical trial for the effect of lithium coupled with prostatectomy on men has been conducted but as of this writing results have not yet been published ${ }^{1}$.

\footnotetext{
${ }^{1}$ https://clinicaltrials.gov/ct2/show/NCT02198859
} 
With respect to other cancers, lithium has been found to be lethal to neuroblastoma cells but not to normal nerve cells (11). A similar effect was found in ovarian cancer cells (12), although a subsequent similar study on ovarian cancer cells suggests only a more modest benefit (13). It is not clear from our reading of the two ovarian cancer papers why the results are significantly different from each other.

With respect to colorectal cancer, one study suggests that lithium inhibits proliferation of a colorectal cancer cell line (14). Another study on colon cancer cells showed that lithium specifically induced a reversal of the epithelial-to-mesenchymal transition characteristic of the cancer cells (15).

Two studies with relatively small sample size suggested a possible link between lithium and tumors of the upper urinary tract $(16,17)$. However, a large-scale study involving all urinary tract cancers in Denmark over a multi-year period found no correlation with lithium use (18).

Because lithium therapy is systemic rather than topical or local, it follows that lithium might inhibit metastasis. Evidence that this is the case for colon cancer comes from observation of inhibition of metastasis-inducing factors by lithium and by observation on reduced metastasis in model animals given lithium therapy (19).

Autophagy is a key cellular process in the inhibition of cancer (20). Lithium has been shown to induce autophagy, due to its inhibition of inositol monophosphatase (21). The full range of lithium effects on autophagy is complicated (22), as might be expected because lithium has multiple targets, which themselves have multiple substrates.

A promising strategy is to combine lithium with other therapies. Many cancer therapies have side effects, so augmentation with lithium may have the dual effect of enhancing the effect of the therapy itself and also permit lower but still effective therapeutic doses. Han et al. (23) reported success in combining lithium with another GSK3 inhibitor against TP53 wild-type glioblastoma cells. Zhukova et al. (24) reported that lithium abrogated TP53-mutant radiation resistance in medulloblastomas, showing that effectiveness of radiation therapy may be enhanced by augmenting with lithium.

Because of the promising indications as cited above, lithium has been suggested as one of a number of drugs commonly used for other reasons, to be repurposed for cancer (25).

\section{Biochemical Context for Lithium and Cancer}

Much of lithium's known biochemical action may be summarized by noting that it inhibits some phosphate-transfer enzymes (primarily phosphatases and kinases) that have magnesium as a co-factor (2). A common underlying biophysical basis for competition between lithium and magnesium for modulating phosphate-transfer enzymes, is suggested by noting that the primary energy source for cells and the substrate for phosphorylating enzymes is not bare ATP, but rather magnesiumassociated ATP (MgATP) (26). NMR studies show that lithium associates with MgATP (27). Based on this admittedly small amount of data, we consider the possibility that lithium generally associates with magnesium-phosphate complexes and thus has the potential to modulate to some extent a large number of phosphorylation reactions and ATP-splitting processes.

Because mutations in $\mathrm{G}$ protein linked receptors have emerged as of interest in cancer research (28), it is significant that lithium appears to inhibit $\beta$-adrenergic and muscarinic receptor coupling to $G$ proteins by competing with magnesium, which facilitates such coupling (29-33).

In the literature we find evidence for direct lithium inhibition of 17 human magnesium-dependent phosphatetransfer enzymes, as follows: A review by Phiel and Klein (34) identified five (IMPase, IPPase, FBPase, BPntase, and GSK3B). Testing against a panel of 80 protein kinases (35) revealed lithium sensitivity for eight more enzymes (MNK1, MNK2, smMLCK, PHK, CHK2, HIPK3, IKK $\epsilon$, and TBK1). It has long been observed that adenyl cyclase activity is inhibited by lithium (36). Of nine different adenylyl cyclases tested, two (ADCY5 and ADCY7) are strongly inhibited by lithium and one (ADCY2) is less strongly but significantly inhibited (37). With the addition of GSK3A (38), we have a list of 17 phosphate-transfer enzymes directly inhibited by lithium. An inspection of protein-protein interaction databases indicates that all 17 interact with multiple other gene products. It should be noted that 72 out of 80 kinases (33), and six out of nine adenylyl cyclases (34), screened were found not to be lithium-sensitive. Table 1 provides the names and synonyms, including both the commonly used gene and protein names, for the 17 known human lithium-sensitive enzymes.

Because lithium affects many different biological molecules and processes (2), it is essential to utilize the tools of systems biology (39) if a comprehensive understanding of lithium action and its prospects for therapy are to be obtained. Important concepts for organizing biological information in a systems context are pathways and networks. A very useful tool for obtaining data about known pathways is the KEGG database (40). An equally useful and complementary tool is the STRING database of interacting proteins (41).

In the present paper we investigate further the possible linkages between lithium and cancer by analyzing the mutual enrichment between STRING-derived interactomes of lithium-sensitive enzymes, and the KEGG pathways associated with cancer.

\section{METHODS}

Analysis was performed on the interactomes of the abovementioned lithium-sensitive proteins. The interactomes of these proteins were extracted from the STRING database of proteinprotein interactions (https://string-db.org). For each key protein, we adjust confidence level and order of neighbors (nearest only or next nearest included), so that each set contains a few hundred proteins. This size is large enough for statistically reliable enrichment analysis.

\section{Disease Association}

We used the R-package KEGGgraph $(42,43)$ to identify the proteins and genes associated with the cancer-relevant pathways. 
TABLE 1 | Names and synonyms for the known human lithium-sensitive enzymes and the genes that code for them, derived from entries in the UniProt database.

\begin{tabular}{ll}
\hline Names and synonyms of human lithium-sensitive & $\begin{array}{l}\text { Functional } \\
\text { class of } \\
\text { enzymes } \\
\text { enzyme }\end{array}$
\end{tabular}

GSK3A, Glycogen synthase kinase-3 alpha

GSK3B, Glycogen synthase kinase-3 beta

Kinase

MNK1, MKNK1, MAP kinase-interacting

Kinase

serine/threonine-protein kinase 1, MAP kinase

Kinase

signal-integrating kinase 1

MNK2, MKNK2, MAP kinase-interacting

serine/threonine-protein kinase 2, MAP kinase

signal-integrating kinase 2, MAPK signal-integrating kinase 2,

GPRK7

smMLCK, Myosin light chain kinase-smooth muscle, MYLK,

Telokin, MLCK

PHK, Phosphorylase b kinase gamma catalytic chain,

PHK-gamma, PHKG2, PSK-C3, Phosphorylase kinase

subunit gamma-2

CHK2, Serine/threonine-protein kinase Chk2, CHEK2, CHK2

checkpoint homolog, Hucds1, hCds1, CDS1, RAD53

HIPK3, Homeodomain-interacting protein kinase 3, Androgen

receptor-interacting nuclear protein kinase, ANPK,

Fas-interacting serine/threonine-protein kinase, FIST, DYRK6,

FIST3, PKY

IKK $\epsilon$, Inhibitor of nuclear factor kappa-B kinase subunit

epsilon, IKBKE, I-kappa-B kinase epsilon, IKK-E, IKK-epsilon,

IkBKE, Inducible I kappa-B kinase, IKK-I, IKKE, IKKI,

KIAA0151

TBK1, Serine/threonine-protein kinase TBK1,

NF-kappa-B-activating kinase, T2K, TANK-binding kinase 1 ,

NAK

IMPase, Inositol monophosphatase 3, IMPAD1, IMP 3,

IMPase 3, Golgi 3-prime phosphoadenosine 5-prime

phosphate 3-prime phosphatase, Golgi-resident PAP

domain-containing protein 1, Inositol-1(or

4)-monophosphatase 3, Myo-inositol monophosphatase $A 3$

IPPase, Inositol polyphosphate 1-phosphatase, IPP, INPP1

FBPase, Fructose-1,6-bisphosphatase 1, FBPase 1,

D-fructose-1,6-bisphosphate 1-phosphohydrolase 1, Liver

FBPase, FBP, FBP1

BPntase, BPNT1, 3' (2'),5'-bisphosphate nucleotidase 1, Bisphosphate $3^{\prime}$-nucleotidase 1, PAP-inositol

1,4-phosphatase, PIP

ADCY2, Adenylate cyclase type 2, ATP pyrophosphate-lyase 2, Adenylate cyclase type II, Adenylyl cyclase 2, KIAA1060

ADCY5, Adenylate cyclase type 5, ATP pyrophosphate-lyase

ADCY7, Adenylate cyclase type 7, ATP pyrophosphate-lyase

7, ATP pyrophosphate-lyase 7, Adenylate cyclase type VII,

Adenylyl cyclase 7, KIAA0037
Phosphatase

Phosphatase

Phosphatase

Adenyl

cyclase

Adenyl

cyclase

Adenyl

cyclase phosphatase, gPAPP, Inositol monophosphatase

5, Adenylate cyclase type V, Adenylate cyclase type V, AC5

gene products (proteins). Our methods and results should be considered in the context of the publication from the American Statistical Association, "The ASA's Statement on $p$ values: Context, Process, and Purpose" (44) In particular the statement notes " $P$-values and related analyses should not be reported selectively. Conducting multiple analyses of the data and reporting only those with certain $p$-values (typically those passing a significance threshold) renders the reported $p$-values essentially uninterpretable." We acknowledge that such selective reporting is common-indeed the ASA statement was prompted in large measure by the widespread practice of such selective reporting. In this paper we will present in graphical form all the $p$-values we compute, so that their significance can be judged in complete context.

For each of the 17 lithium sets, an ensemble of 1,000 null sets are generated by random selection from the human genome. Each null set is the same size as the corresponding lithium set. Then we used the R-package STRINGdb (45) to perform KEGG pathway enrichment analysis. This operation is a particular example of the powerful technique of gene-annotation enrichment analysis (46). In gene-annotation enrichment analysis a test list of genes (often derived from gene expression experiments) or proteins is compared to an organized database of gene annotations, often referred to as a gene ontology (47), an array of gene lists corresponding to different biological functions, molecular functions, or locations in the cell. Although the phrase "gene ontology" is used to describe the database, in fact the objects in the database are the gene products, or proteins, whose biological function, molecular function, and location in cell are tagged with annotations. The output of the gene/proteinannotation enrichment analysis is expressed as the likelihood that the list overlaps could have occurred by chance ( $p$-value). A very low $p$-value implies that the degree of overlap is highly significant statistically and very likely is significant biologically. In our study the lists we are comparing are the proteins contained in the interactomes of lithium sensitive enzymes on the one hand, and the proteins and genes contained in the KEGG pathways associated with cancer on the other hand. For each KEGG term retrieved, a null distribution of uncorrected $p$-value is generated by the 1,000 null sets. This gives us a measure of the false discovery rate, since any overlap between the null sets and the KEGG pathways is purely accidental. Then the fraction of null set uncorrected $p$-values smaller than or equal to the lithiumsensitive set uncorrected $p$-value would be the empirical $p$-value. A flow chart for this calculation is provided in Figure 1. For a detailed discussion of empirical $p$-value determination see Ge et al. (48).

\section{RESULTS}

\section{$\boldsymbol{P}$-Value Calculation}

The fundamental question we address is whether there is significant overlap or mutual enrichment between the interactomes of lithium-sensitive proteins and the pathways implicated in various cancers. Both the interactomes and the pathways are represented by lists of their constituent
Figure 2 shows mutual lithium interactome enrichment with specific cancer pathways, represented by heatmaps. Each area on the heatmap is a color-coded representation of the degree of mutual enrichment between the genes in the interactome of the indicated lithium sensitive enzyme and the genes in the indicated pathway. The darker the shade, the more significant the mutual 


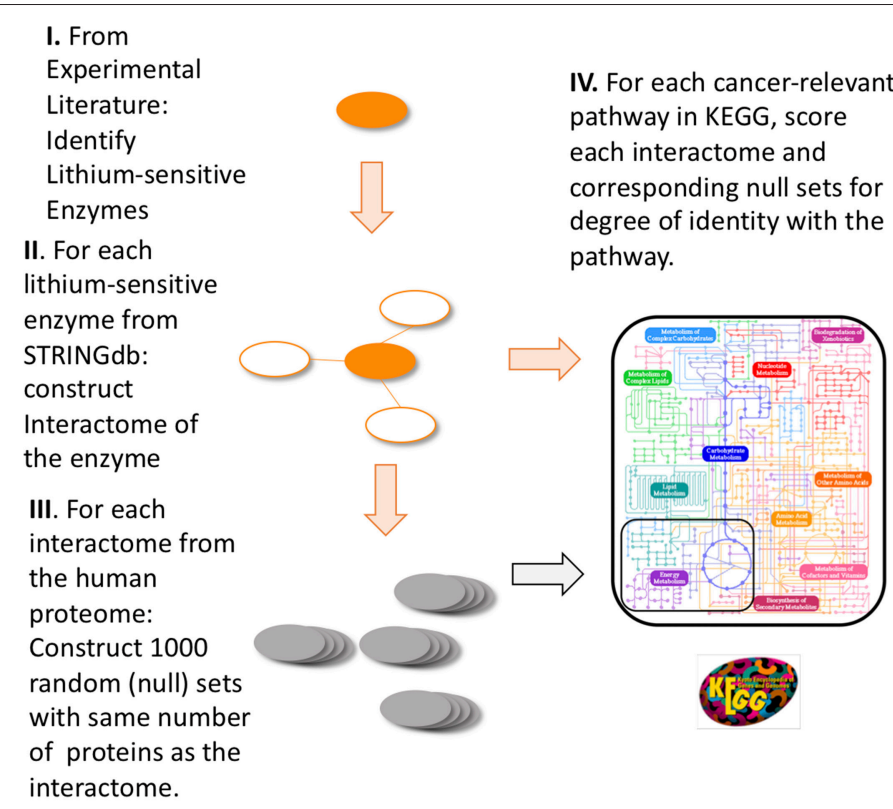

\section{For each interactome-pathway pair: Tabulate the number of times that a null set has an equal or greater degree of identity with the pathway as the interactome and divide by 1000 . This is the $p$-value of the mutual enrichment between the interactome and the pathway.}

FIGURE 1 | Flow diagram of the steps to compute empirical $p$-values for the mutual enrichment between the interactomes of lithium-sensitive enzymes and cancer-relevant pathways. The steps are: (1) From the experimental literature, identify the lithium-sensitive enzymes, (2) using the STRING database, construct the interactome of each of the lithium-sensitive enzymes, (3) For each interactome, construct 1,000 null sets consisting of proteins randomly chosen from the entire human proteome with each null set containing the same number as the interactome, (4) for each interactome and null set, calculate the degree of identity with the list of proteins from each cancer-relevant pathway, (5) for each interactome-pathway combination, tabulate the number of times the null set has equal or greater degree of identity with the pathway set than does the interactome. This number, divided by 1,000 , is the $p$-value for mutual enrichment between the interactome and the pathway.

enrichment of the interactome-pathway combination is. The light areas on the heatmap represent situations where a lithiumsensitive interactome has little or no mutual enrichment with a cancer pathway. The dark areas, deep orange and red, represent situations where enrichment is very strong-far greater than could be expected by chance and therefore statistically highly significant. The deep red color between $1 \mathrm{E}-3$ and $1 \mathrm{E}-4$ on the vertical scale is uniform and represents the situation where not even one of the 1,000 null sets was as mutually enriched with the pathway as was the interactome.

It appears that the interactomes of five out of the 17 lithiumsensitive genes (ADCY2, ADCY5, ADCY7, BPNT1, and HIPK3) do not show significant mutual enrichment with the cancer pathways explored in this study. Chemical carcinogenesis shows significant mutual enrichment with only one of the interactomes, that of IMPAD1. For the remaining specific cancer pathways and lithium-sensitive interactomes, there are multiple areas of strong mutual enrichment, as indicated by deep orange to red coloring. The genes contained in these overlapping areas, and their modes of regulation, appear worthy of further study in unraveling the details of the lithium vs. cancer relationship.

In addition to the labeled specific cancer pathways we extended the analysis to signaling pathways in which dysfunction is implicated in cancer, as indicated in the literature (49-56). Figure 3 shows in heatmap form the mutual enrichment between the 17 lithium-sensitive interactomes and 13 pathways relevant to cancer. In Figure 3 we find that the interactomes of the lithium sensitive enzymes ADCY2, ADCY5, ADCY7, and BPNT1 that did not show strong enrichment in any of the pathways for specific cancers do in fact show strong enrichment with some cancer-relevant signaling pathways. Specifically, ADCY2, ADCY5, and ADCY7 show strong enrichment with the Ras signaling pathway and BPNT1 shows strong enrichment with the Notch signaling pathway. Only HIPK3 remains without strong enrichment with any relevant pathway.

The inescapable conclusion from Figures 2, 3 is that variability in lithium concentration is likely to significantly modulate most cancer-relevant pathways. We should note that sensitivity to lithium does not necessarily imply a beneficial sensitivity. There are some indications for some cancers that lithium might be beneficial, as described in the Introduction section of this paper, but because of the complexity of the feedback relationships in these pathways, a complicated relationship between lithium ingestion and cancer incidence is very possible.

\section{SUMMARY AND DISCUSSION}

We have conducted a pathway and network enrichment analysis exploring the role of lithium in multiple cancers and cancerrelated pathways. The results show that for the large majority of such cancers, there is high mutual enrichment between the interactomes of lithium-sensitive enzymes and the pathways associated with those diseases, indicating that lithium is very 


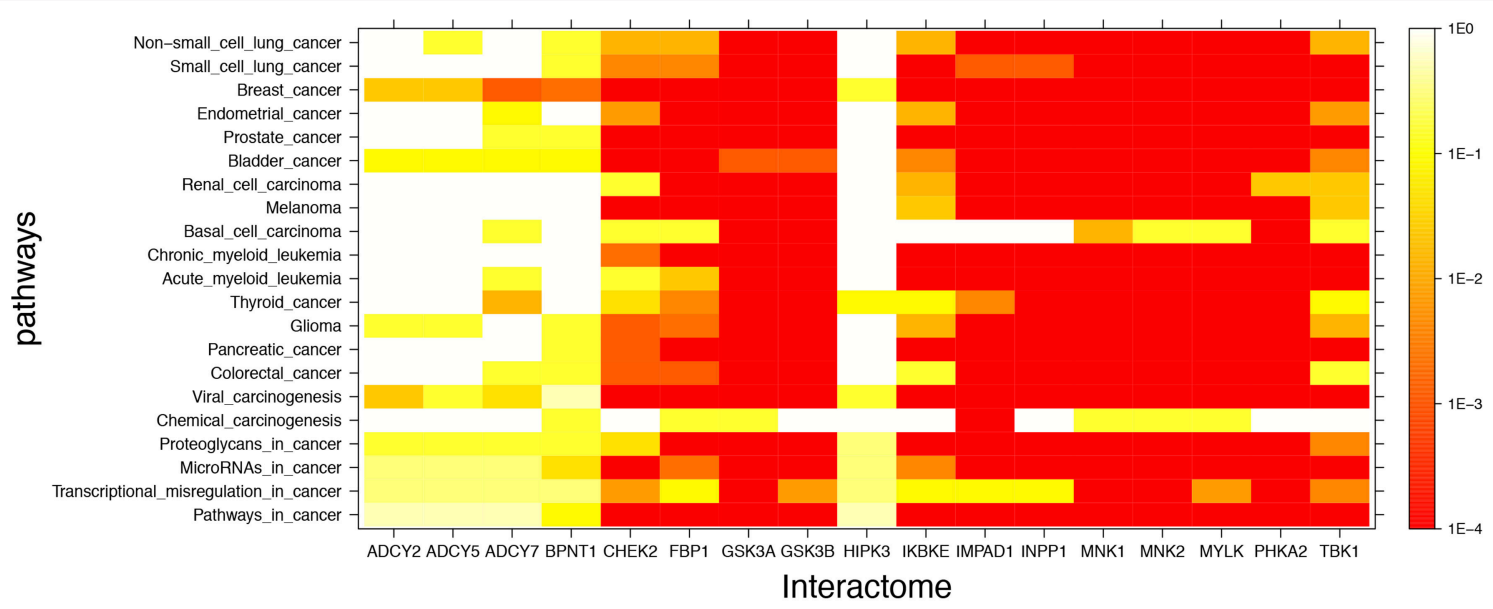

FIGURE 2 | Visual representation of mutual enrichment patterns between specific cancer pathways and the interactomes of lithium-sensitive gene products. Calibration of $p$-value vs. color is indicated by a vertical scale to the right of the heat map. Red or dark orange indicates very strong enrichment while lighter color indicates weak or, if white, no enrichment. Five genes stand out as being not strongly connected to these cancer pathways: BPNT1, HIPK3, ADCY2, ADCY5, and ADCY7. Of the cancer pathways, chemical carcinogenesis stands out as being less likely to be strongly influenced by lithium levels, although there is a strong mutual enrichment between the interactome of IMPAD1 and this pathway. For the remainder of the genes and the remainder of the cancers, the relationship between the lithium-sensitive interactome and the cancer phenotype is strong.

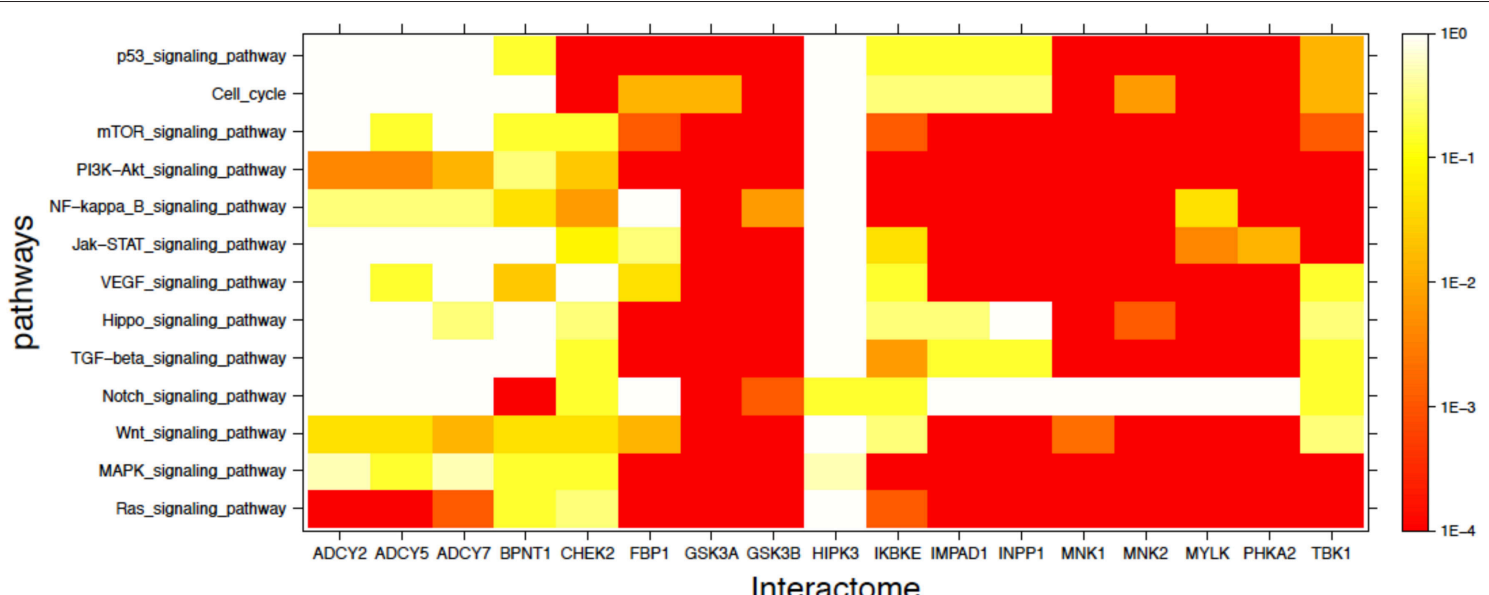

FIGURE 3 | Visual representation of mutual enrichment patterns between signaling pathways implicated in cancer and the interactomes of lithium-sensitive gene products. Calibration of $p$-value vs. color is indicated by a vertical scale to the right of the heat map. Red or dark orange indicates very strong enrichment while lighter color indicates weak or, if white, no enrichment. Only one gene product appears not relevant to cancer, HIPK3. The three adenyl cyclases, BPNT1, and CHEK2 show strong mutual enrichment for a couple of the pathways. Each of the remaining 11 interactomes show strong mutual enrichment with most of the cancer-relevant pathways.

likely to affect the incidence and course of the disease. Our results are consistent with a variety of lines of evidence from both epidemiology and from experiment, cited in earlier sections of this paper, suggesting possible influence of lithium on the incidence and progression of cancer.

We hope that the results described in this paper will contribute to prioritizing and designing clinical trials of lithium for cancer. To provide context for such prioritization and design, it is essential to take into account the ways in which lithium is unique, both as a pharmaceutical and as an ion that is ubiquitous in the environment, and therefore ubiquitous in the water and food we ingest (2):

1. Unlike other ions, lithium is not closely regulated by selective membrane transport processes. Rather it shares transport and permeation pathways that are mainly selective for other ions, in most cases sodium (2). Therefore, lithium concentration in both extracellular and intracellular compartments, rather than being nearly constant as is the case with other ions, is roughly proportional to lithium ingestion (57). Whereas, changes in the concentrations of 
other ions of more than a few percent have severe acute adverse consequences, the human body adjusts without acute adverse consequence to changes in lithium concentrations of several orders of magnitude. Our biochemistry has evolved to accommodate to widely varying lithium levels, as opposed to developing the ability to closely regulate lithium levels.

2. The multiple enzymes inhibited by lithium are each functionally linked to large numbers of other genes. This explains why the effects of lithium are widespread and varied; lithium has a modulating effect on many gene networks. We note that screening for lithium sensitivity has so far not included systematic examination of multiple variants of particular gene products, either mutational variants or alternative splices from the same gene. Therefore, it may be that some of the enzymes that have been found not lithium-sensitive may have mutational or splice variants that are sensitive. Conversely, some of the enzymes that have been found to be lithium-sensitive may have mutational or splice variants that are insensitive. The plausibility of such a possibility is exemplified by a functional, structural, and mutational study on an archaeal inositol monophosphatase (58). The archaeal enzyme has high homology (30\% identical, $50 \%$ similar) to its human counterpart and functions in the same magnesium-dependent manner. In this study it was shown that a single amino acid substitution could convert the enzyme from its native lithium-insensitive form to a lithium-sensitive form. Perhaps of relevance, it has long been known that lithium responsiveness is significantly variable among human individuals (59).

3. Unlike other pharmaceuticals, lithium is probably an essential trace element in the diet (60-62). The question with lithium is not whether it should be ingested or not, but rather how much. Extreme lithium deprivation results in failure to thrive, while too much lithium is toxic. The existence of these extrema suggests existence of an intermediate optimum.

Therefore, we suggest that the correct question to ask with respect to lithium and a particular disease is not, "Should lithium be administered for this particular disease?" but rather, "What is the optimum blood level of lithium for this individual, given his or her disease history, status, genetic propensities, and other medications?" Unlike some pharmaceuticals that are more specific and inhibit or activate one gene or a small number of genes, the model for lithium action is that it alters the balance between a large number of interacting processes and pathways. Thus, a dose-response curve for lithium is likely to be highly non-linear and not always monotonic.

There are just a few well-established markers for optimum concentrations. For a patient with a reliable diagnosis of bipolar disorder a common target for optimality would be blood concentration of $0.8-1 \mathrm{mM}$. Significantly higher concentrations

\section{REFERENCES}

1. Post RM. Treatment of bipolar depression: evolving recommendations. Psychiatr Clin. (2016) 9:11-33. doi: 10.1016/j.psc.2015.09.001 will result in acute toxicity, while significantly lower will result in loss of effectiveness. However, this level has some side effects when sustained for years or decades, namely an increased risk of kidney damage and lowered thyroid activity (63).

At the other end of the dosage scale, epidemiological evidence is compelling that geographical variations in concentration of lithium in the drinking water are correlated with a variety of health and wellness markers, most notably and reliably with incidence of suicide (64-69).

Another important marker is provided by a study showing that over a 4 -year period a lithium level of $0.25-0.4 \mathrm{mM}$ of lithium ( $1 / 4$ to $1 / 2$ of the bipolar therapeutic dose) did not incur any renal damage (70). This study suggests that clinical studies exploring low to medium-dose lithium could be undertaken with relatively minimal concerns for side effects.

One possible piece of low-hanging fruit for a clinical trial would be low- to medium-dose lithium for men undergoing active surveillance (AS) for advance of prostate cancer. From studies of AS outcomes, a large fraction of patients on AS ultimately require invasive treatment, as reviewed by Dall'Era et al. (71). When this need arises it typically comes after only a few years. Thus, a trial of lithium in this context would produce significant results in a short time and would be relatively inexpensive.

A second area that seems ready for clinical trial is augmentation of other cancer therapies, either radiation or pharmaceutical, with low-to-moderate lithium. Studies we have cited in this paper support the possibility of beneficial results, and also support the lack of side effects from such lithium doses.

\section{AUTHOR CONTRIBUTIONS}

The work was planned jointly in conversations between EJ and WG. WG did the computations and prepared the figures and tables. WG wrote the first draft of the Methods and Results sections. EJ wrote the first draft of the Introduction and Conclusions sections. Both authors shared in the final refinement of the manuscript.

\section{FUNDING}

Salary support for WG by National Science Foundation grant 1624615 is gratefully acknowledged.

\section{ACKNOWLEDGMENTS}

We gratefully acknowledge very useful comments from Peter Klein on an earlier version of this manuscript, and from the reviewers of this paper, whose comments have resulted in improvements. An earlier version of this work was posted on the preprint server bioRxiv (72).

2. Jakobsson E, Argüello-Miranda O, Chiu S, Fazal Z, Kruczek J, Nunez-Corrales $\mathrm{S}$, et al. Towards a unified understanding of lithium action in basic biology and its significance for applied biology. J Membr Biol. (2017) 250:587-604. doi: 10.1007/s00232-017-9998-2 
3. Ge W, Jakobsson E. Systems biology understanding of the effects of lithium on affective and neurodegenerative disorders. Front Neurosci. (2018) 12:933. doi: 10.3389/fnins.2018.00933

4. Cohen Y, Chetrit A, Sirota P, Modan, B. Cancer morbidity in psychiatric patients: influence of lithium carbonate treatment. Med Oncol. (1998) 15:32-6. doi: $10.1007 / \mathrm{BF} 02787342$

5. Martinsson L, Westman J, Hällgren J, Ösby U, Backlund L. Lithium treatment and cancer incidence in bipolar disorder. Bipolar Disord. (2016) 18:33-40. doi: 10.1111/bdi.12361

6. Huang R-Y, Hsieh K-P, Huang W-W, Yang Y-H. Use of lithium and cancer risk in patients with bipolar disorder: population-based cohort study. $\mathrm{Br} J$ Psychiatry. (2016) 209:393-9. doi: 10.1192/bjp.bp.116.181362

7. Pottegård A, Ennis ZN, Hallas J, Jensen BL, Madsen K, Friis S. Long-term use of lithium and risk of colorectal adenocarcinoma: a nationwide case-control study. Br J Cancer. (2016) 114:571-5. doi: 10.1038/bjc.2016.10

8. Mazor M, Kawano Y, Zhu H, Waxman J, Kypta RM. Inhibition of glycogen synthase kinase-3 represses androgen receptor activity and prostate cancer cell growth. Oncogene. (2004) 23:7882. doi: 10.1038/sj.onc.1208068

9. Sun A, Shanmugam I, Song J, Terranova PF, Thrasher JB, Li B. Lithium suppresses cell proliferation by interrupting E2F-DNA interaction and subsequently reducing S-phase gene expression in prostate cancer. Prostate. (2007) 67:976-88. doi: 10.1002/pros.20586

10. Qing Z, Yang J, Han S, Liu J, Holzbeierlein J, Thrasher JB, et al. Suppression of glycogen synthase kinase 3 activity reduces tumor growth of prostate cancer in vivo. Prostate. (2011) 71:835-45. doi: 10.1002/pros.21300

11. Duffy DJ, Krstic A, Schwarzl D, Higgins DG, Kolch W. GSK3 inhibitors regulate MYCN mRNA levels and reduce neuroblastoma cell viability through multiple mechanisms, including p53 and Wnt signaling. Mol Cancer Ther. (2014) 13:454-67. doi: 10.1158/1535-7163.MCT-13-0560-T

12. Cao Q, Lu X, Feng Y-J. Glycogen synthase kinase- $3 \beta$ positively regulates the proliferation of human ovarian cancer cells. Cell Res. (2006) 16:671-7. doi: $10.1038 /$ sj.cr.7310078

13. Novetsky AP, Thompson DM, Zighelboim I, Thaker PH, Powell MA, Mutch DG, et al. Lithium and inhibition of GSK3 $\beta$ as a potential therapy for serous ovarian cancer. Int J Gynecol Cancer. (2013) 23:361. doi: 10.1097/IGC.0b013e31827cfecb

14. Li H, Huang K, Liu X, Liu J, Lu X, Tao K, et al. Lithium chloride suppresses colorectal cancer cell survival and proliferation through ROS/GSK-3 $\beta / \mathrm{NF}$ КB signaling pathway. Oxidat Med Cell Longevity. (2014) 2014:241864. doi: 10.1155/2014/241864

15. Costabile V, Duraturo F, Delrio P, Rega D, Pace U, Liccardo R, et al. Lithium chloride induces mesenchymal to epithelial reverting transition in primary colon cancer cell cultures. Int J Oncol. (2015) 46:1913-23. doi: 10.3892/ijo.2015.2911

16. Rookmaaker MB, van Gerven HAJM, Goldschmeding R, Boer WH. Solid renal tumours of collecting duct origin in patients on chronic lithium therapy. Clin Kidney J. (2012) 5:412-5. doi: 10.1093/ckj/sfs091

17. Zaidan M, Stucker F, Stengel B, Vasiliu V, Hummel A, Landais P, et al. Increased risk of solid renal tumors in lithium-treated patients. Kidney Int. (2014) 86:184-90. doi: 10.1038/ki.2014.2

18. Pottegård A, Hallas J, Jensen BL, Madsen K, Friis S. Long-term lithium use and risk of renal and upper urinary tract cancers. J Am Soc Nephrol. (2015) 27:249-55. doi: 10.1681/ASN.2015010061

19. Maeng Y-S, Lee R, Lee B, Choi S-I, Kim FA. Lithium inhibits tumor lymphangiogenesis and metastasis through the inhibition of TGFBIp expression in cancer cells. Sci Rep. (2016) 6:20739. doi: 10.1038/srep20739

20. Zhong Z, Sanchez-Lopez E, Karin M. Autophagy, inflammation, and immunity: a troika governing cancer and its treatment. Cell. (2016) 166:28898. doi: 10.1016/j.cell.2016.05.051

21. Sovan S, Floto RA, Berger Z, Imarisio S, Cordenier A, Pasco M, et al. Lithium induces autophagy by inhibiting inositol monophosphatase. J Cell Biol. (2005) 170:1101-11. doi: 10.1083/jcb.200504035

22. Yumiko M, Shimada K, Ishiguro K, Hattori N. Lithium and autophagy. ACS Chem Neurosci. (2014) 5:434-42. doi: 10.1021/cn500056q

23. Han S, Meng L, Jiang Y, Cheng W, Tie, Xia XJ, et al. Lithium enhances the antitumour effect of temozolomide against TP53 wild-type glioblastoma cells via NFAT1/FasL signalling. Br J Cancer. (2017) 116:1302. doi: 10.1038/bjc.2017.89
24. Zhukova N, Ramaswamy V, Remke M, Martin DC, Castelo-Branco P, Zhang $\mathrm{CH}$, et al. WNT activation by lithium abrogates TP53 mutation associated radiation resistance in medulloblastoma. Acta Neuropathol Commun. (2014) 2:174. doi: 10.1186/s40478-014-0174-y

25. Sleire L, Førde-Tislevoll HE, Netland IA, Leiss L, Skeie BS, Enger PØ. Drug repurposing in cancer. Pharmacol Res. (2017) 124:74-91. doi: 10.1016/j.phrs.2017.07.013

26. Gout E, Rébeillé F, Douce R, Bligny R. Interplay of $\mathrm{Mg} 2+, \mathrm{ADP}$, and ATP in the cytosol and mitochondria: unravelling the role of $\mathrm{Mg} 2+$ in cell respiration. Proc Natl Acad Sci USA. (2014) 111:E4560-7. doi: 10.1073/pnas.1406251111

27. Briggs KT, Giulian GG, Li G, Kao JPY, Marino JP. A molecular model for lithium's bioactive form. Biophys J. (2016) 111:294-300. doi: 10.1016/j.bpj.2016.06.015

28. O’hayre M, Vázquez-Prado J, Kufareva I, Stawiski EW, Handel TM, Seshagiri S, et al. The emerging mutational landscape of $\mathrm{G}$ proteins and G-protein-coupled receptors in cancer. Nat Rev Cancer. (2013) 13:412. doi: 10.1038/nrc3521

29. Avissar S, Murphy DL, Schreiber G. Magnesium reversal of lithium inhibition of $\beta$-adrenergic and muscarinic receptor coupling to $G$ proteins. Biochem Pharmacol. (1991) 41:171-5. doi: 10.1016/0006-2952(91)9 0473-I

30. Mota de Freitas D, Castro MMCA, Geraldes CFGC. Is competition between $\mathrm{Li}+$ and $\mathrm{Mg} 2+$ the underlying theme in the proposed mechanisms for the pharmacological action of lithium salts in bipolar disorder? Accounts Chem Res. (2006) 39:283-91. doi: 10.1021/ar030197a

31. Yoshikawa T, Honma S. Lithium lengthens circadian period of cultured brain slices in area specific manner. Behav Brain Res. (2016) 314:30-7. doi: 10.1016/j.bbr.2016.07.045

32. Bauer M, Gitlin M. What is lithium and how does it work? In: The Essential Guide to Lithium Treatment. Springer International Publishing (2016). p. 33-43. doi: 10.1007/978-3-319-31214-9_4

33. Wang H-Y, Friedman E. Effects of lithium on receptor-mediated activation of G proteins in rat brain cortical membranes. Neuropharmacology. (1999) 38:403-14. doi: 10.1016/S0028-3908(98)00197-X

34. Phiel CJ, Klein PS. Molecular targets of lithium action. Annu Rev Pharmacol Toxicol. (2001) 41:789-813. doi: 10.1146/annurev.pharmtox.41.1.789

35. Bain J, Plater L, Elliott M, Shpiro N, Hastie CJ, Mclauchlan H, et al. The selectivity of protein kinase inhibitors: a further update. Biochem J. (2007) 408:297-315. doi: 10.1042/BJ20070797

36. Forn J, Valdecasas FG. Effects of lithium on brain adenyl cyclase activity. Biochem Pharmacol. (1971) 20:2773-9. doi: 10.1016/0006-2952(71)9 0187-0

37. Mann L, Heldman E, Shaltiel G, Belmaker RH, Agam G. Lithium preferentially inhibits adenylyl cyclase V and VII isoforms. Int J Neuropsychopharmacol. (2008) 11:533-9. doi: 10.1017/S1461145707008395

38. Freland L, Beaulieu J-M. Inhibition of GSK3 by lithium, from single molecules to signaling networks. Front Mol Neurosci. (2012) 5:14. doi: $10.3389 /$ fnmol.2012.00014

39. Kitano H. Systems biology: a brief overview. Science. (2002) 295:1662-4. doi: 10.1126/science.1069492

40. Kanehisa M, Furumichi M, Tanabe M, Sato Y, Morishima K. KEGG: new perspectives on genomes, pathways, diseases and drugs. Nucleic Acids Res. (2016) 45:D353-61. doi: 10.1093/nar/gkw1092

41. Szklarczyk D, Morris JH, Cook H, Kuhn M, Wyder S, Simonovic M, et al. The STRING database in 2017: quality-controlled protein-protein association networks, made broadly accessible. Nucleic Acids Res. (2016) 2016:gkw937. doi: 10.1093/nar/gkw937

42. Zhang JD, Wiemann S. KEGGgraph: a graph approach to KEGG PATHWAY in R and Bioconductor. Bioinformatics. (2009) 25:1470-1. doi: 10.1093/bioinformatics/btp167

43. Zhang JD. KEGGgraph: Application Examples. $\mathrm{R}$ package version 1.38.0 (2017).

44. Wasserstein RL, Nicole A, Lazar NA. The ASA's statement on pvalues: context, process, and purpose. Am Stat. (2016) 70:129-33. doi: 10.1080/00031305.2016.1154108

45. Franceschini A. STRING v9.1: protein-protein interaction networks, with increased coverage and integration. Nucleic Acids Res. (2013) 41:D808-15. doi: 10.1093/nar/gks1094 
46. Huang DW, Sherman BT, Richard A, Lempicki RA. Bioinformatics enrichment tools: paths toward the comprehensive functional analysis of large gene lists. Nucleic Acids Res. (2008) 37:1-13. doi: 10.1093/nar/gkn923

47. Thomas PD. Expansion of the gene ontology knowledgebase and resources: the gene ontology consortium. Nucleic Acids Res. (2017) 45:D331-8. doi: 10.1093/nar/gkw1108

48. Ge, W, Fazal Z, Jakobsson E. Using optimal F-measure and random resampling in gene ontology enrichment calculations. bioRxiv. (2017) 2017:218248. doi: 10.1101/218248

49. Sanchez-Vega F, Mina M, Armenia J, Chatila WK, Luna A, La KC, et al. Oncogenic signaling pathways in the cancer genome atlas. Cell. (2018) 173:321-37. doi: 10.1016/j.cell.2018.03.035

50. Thomas SJ, Snowden JA, Zeidler MP, Danson SJ. The role of JAK/STAT signalling in the pathogenesis, prognosis and treatment of solid tumours. $\mathrm{BrJ}$ Cancer. (2015) 113:365. doi: 10.1038/bjc.2015.233

51. Kieran MW, Kalluri R, Cho Y-J. The VEGF pathway in cancer and disease: responses, resistance, and the path forward. Cold Spring Harbor Perspect Med. (2012) 2012:a006593. doi: 10.1101/cshperspect.a0 06593

52. Ferrara N. VEGF and the quest for tumour angiogenesis factors. Nat Rev Cancer. (2002) 2:795. doi: 10.1038/nrc909

53. Zeligs KP, Neuman MK, Annunziata CM. Molecular pathways: the balance between cancer and the immune system challenges the therapeutic specificity of targeting nuclear factor- $\kappa \mathrm{b}$ signaling for cancer treatment. Clin Cancer Res. (2016) 22:4302-8. doi: 10.1158/1078-0432.CCR-15-1374

54. Xavier D, Llobet D, Pallares J, Matias-Guiu X. NF-kB in development and progression of human cancer. Virchows Arch. (2005) 446:475-82. doi: 10.1007/s00428-005-1264-9

55. Guertin DA, Sabatini DM. Defining the role of mTOR in cancer. Cancer Cell. (2007) 12:9-22. doi: 10.1016/j.ccr.2007.05.008

56. Dhillon AS, Hagan S, Rath O, Kolch W. MAP kinase signalling pathways in cancer. Oncogene. (2007) 26:3279. doi: 10.1038/sj.onc.1210421

57. de Roos NM, de Vries JHM, Katan MB. Serum lithium as a compliance marker for food and supplement intake. Am J Clin Nutr. (2001) 73:75-9. doi: 10.1093/ajcn/73.1.75

58. Zheng L, Stieglitz KA, Shrout AL, Wei Y, Weis RM, Stec B, et al. Mobile loop mutations in an archaeal inositol monophosphatase: modulating three-metal ion assisted catalysis and lithium inhibition. Protein Sci. (2010) 19:309-18. doi: 10.1002/pro.315

59. Liping H, Heilbronner U, Degenhardt F, Adli M, Akiyama K, Akula N, et al. Genetic variants associated with response to lithium treatment in bipolar disorder: a genome-wide association study. Lancet. (2016) 387:1085-93. doi: 10.1016/S0140-6736(16)00143-4

60. Anke M, Arnhold W, Groppel B, Krause U. The biological importance of lithium. Lithium Biol Med. (1991) 1991:149-67.
61. Pickett EE, O'Dell BL. Evidence for dietary essentiality of lithium in the rat. Biol Trace Element Res. (1992) 34:299-319. doi: 10.1007/BF02783685

62. Schrauzer GN. Lithium: occurrence, dietary intakes, nutritional essentiality. J Am Coll Nutr. (2002) 21:14-21. doi: 10.1080/07315724.2002.10719188

63. McKnight RF, Adida M, Budge K, Stockton S, Goodwin GM, Geddes JR. Lithium toxicity profile: a systematic review and meta-analysis. Lancet. (2012) 379:721-8. doi: 10.1016/S0140-6736(11)61516-X

64. Schrauzer GN, Shrestha KP. Lithium in drinking water and the incidences of crimes, suicides, and arrests related to drug addictions. Biol Trace Element Res. (1990) 25:105-13. doi: 10.1007/BF02990271

65. Ohgami H, Terao T, Shiotsuki I, Ishii N, Iwata N. Lithium levels in drinking water and risk of suicide. Br J Psychiatry. (2009) 194:464-5. doi: 10.1192/bjp.bp.108.055798

66. Helbich M, Leitner M, Kapusta ND. Geospatial examination of lithium in drinking water and suicide mortality. Int J Health Geogr. (2012) 11:19. doi: 10.1186/1476-072X-11-19

67. Giotakos O, Nisianakis P, Tsouvelas G, Giakalou V-V. Lithium in the public water supply and suicide mortality in Greece. Biol Trace Element Res. (2013) 156:376-9. doi: 10.1007/s12011-013-9815-4

68. Blüml V, Regier MD, Hlavin G, Rockett IRH, König F, Vyssoki B, et al. Lithium in the public water supply and suicide mortality in Texas. J Psychiatr Res. (2013) 47:407-11. doi: 10.1016/j.jpsychires.2012.12.002

69. Kapusta ND, Mossaheb N, Etzersdorfer E, Hlavin G, Thau K, Willeit M, et al. Lithium in drinking water and suicide mortality. Br J Psychiatry. (2011) 198:346-50. doi: 10.1192/bjp.bp.110.091041

70. Aprahamian I, Santos FS, dos Santos B, Talib L, Diniz BS, Radanovic M, et al. Long-term, low-dose lithium treatment does not impair renal function in the elderly: a 2-year randomized, placebo-controlled trial followed by single-blind extension. J Clin Psychiatry. (2014) 75:672-8. doi: 10.4088/JCP.13m08741

71. Dall'Era MA, Albertsen PC, Bangma C, Carroll PR, Carter HB, Cooperberg $\mathrm{MR}$, et al. Active surveillance for prostate cancer: a systematic review of the literature. Eur Urol. (2012) 62:976-83. doi: 10.1016/j.eururo.2012.05.072

72. Ge W, Jakobsson E. Systems biology understanding of the effects of lithium on cancer. bioRxiv. (2018) 2018:363077. doi: 10.1101/363077

Conflict of Interest Statement: The authors declare that the research was conducted in the absence of any commercial or financial relationships that could be construed as a potential conflict of interest.

Copyright (c) 2019 Ge and Jakobsson. This is an open-access article distributed under the terms of the Creative Commons Attribution License (CC BY). The use, distribution or reproduction in other forums is permitted, provided the original author(s) and the copyright owner(s) are credited and that the original publication in this journal is cited, in accordance with accepted academic practice. No use, distribution or reproduction is permitted which does not comply with these terms. 